\title{
The Number of Lattice Points in a k-dimensional Hypersphere ${ }^{*}$
}

\author{
By W. C. Mitchell
}

1. Introduction. One of the most interesting problems of analytic number theory involves the difference between the number of lattice points in a $k$-dimensional hypersphere and the "volume" of the hypersphere. Define the set $L_{k}(x)$ as follows:

$$
L_{k}(x)=\left\{\left(J_{1}, J_{2}, \cdots, J_{k}\right) \mid \sum_{i=1}^{k} J_{i}^{2} \leqq x\right\}
$$

where the $J_{i}$ are integers. Let $A_{k}(x)$ be the number of distinct points in $L_{k}(x)$. Thus $A_{k}(x)$ is the number of lattice points in a $k$-dimensional hypersphere of radius $x^{1 / 2}$. Define $V_{k}(x)$ as the "volume" of a $k$-dimensional hypersphere of radius $x^{1 / 2}$.

$$
V_{k}(x)=\frac{\pi^{k / 2} x^{k / 2}}{\Gamma\left(\frac{k}{2}+1\right)}=\frac{\pi^{[k / 2]} x^{k / 2}}{\frac{k}{2}\left(\frac{k}{2}-1\right) \cdots\left(1 \text { or } \frac{1}{2}\right)}
$$

where $[z]$ is the integer part of $z$.

The problem of primary interest is to find the Greatest Lower Bound $\theta_{k}$ of the set of values $\theta$ for which

$$
P_{k}(x) \equiv A_{k}(x)-V_{k}(x)=O\left(x^{\theta}\right) .
$$

Walfisz [1] gives the following general results:

$$
\begin{aligned}
& P_{k}(x)=O\left(x^{(k-1) / 2}\right), \quad P_{k}(x)=\Omega\left(x^{k / 2-1}\right), \\
& P_{4}(x)=O\left(x \log ^{2} x\right)=O\left(x^{1+\epsilon}\right), \quad \epsilon>0, \\
& P_{k}(x)=O\left(x^{k / 2-1}\right), \quad k \geqq 5 .
\end{aligned}
$$

Thus for $k \geqq 4 \theta_{k}=k / 2-1$.

The value of $k$ which has received the greatest attention is $k=2$, the number of lattice points in a circle. Wilton [2] gives an account of the early work in this problem. Since that time several results have been published establishing new values of $\theta$ for which $P_{2}(x)=O\left(x^{\theta}\right)$. One of the most recent is Chen Jing-ren's proof [3] that $P_{2}(x)=O\left(x^{12 / 37}\right)$. Hardy (see [2]) has shown that $P_{2}(x)=\Omega\left(x^{1 / 4}\right)$. It is a common conjecture that $P_{2}(x)=O\left(x^{1 / 4+\epsilon}\right), \epsilon>0$, or $\theta_{2}=\frac{1}{4}$.

There is less known for $k=3$. From (4) we have $\frac{1}{2} \leqq \theta_{3} \leqq 1$. Fraser and Gotlieb [4] conjectured on the basis of numerical evidence that $.5 \leqq \theta_{3} \leqq .7$. More recently Chen Jing-ren [5] has shown that $\frac{1}{2} \leqq \theta_{3} \leqq \frac{2}{3}$.

With the advent of high speed computers it has become possible to evaluate

Received April 8, 1965.

* This work began with the support of a National Science Foundation grant to the Digital Computer Laboratory of the University of Illinois for the summer of 1964 and was continued with some financial support for auxiliary computing time from the Computing Center of Miami (Ohio) University and from the Department of Mathematics of the California Institute of Technology. 
$P_{k}(x)$ for "large" $x$ in order to see if the calculated results are consistent with theoretical results or if it is reasonable to make any new conjectures concerning $\theta_{k}$. There have been at least three previous papers on this subject. Fraser and Gotlieb. [4] calculated isolated values of $P_{2}(x)$ and $P_{3}(x)$ for $x^{1 / 2}<2000$ on an IBM 650 . However their conclusions differ with the present paper for $\theta_{2}$. Harry Mitchell [6] calculated $P_{2}(x)$ for $x^{1 / 2} \leqq 200,000$ on an IBM 7090, but the results for $x^{1 / 2} \geqq 3000$ are incorrect, as pointed out by Keller and Swenson [7]. Keller and Swenson determined $P_{2}(x)$ for many values of $x^{1 / 2}<260,000$ on an IBM 7090 and their method of interpretation leads them to suggest that $\theta_{2} \leqq .3$. This seems unlikely from the results of the present method of interpretation.

The problem of establishing that $P_{k}(x)=O\left(x^{\theta}\right)$ is equivalent to finding a sequence $\left\langle\left(X_{i}, Y_{i}\right)\right\rangle_{i=1}^{\infty}$ such that

$$
\left|P_{k}(x)\right| \leqq Y_{i}+O\left(x^{\theta}\right) \text { for } x \leqq X_{i+1} \text { and } \limsup _{i \rightarrow \infty} \frac{Y_{i}}{X_{i}{ }^{\theta}}<\infty .
$$

Since $L_{k}(x)$ is composed only of $k$-tuples of integers, $A_{k}(x)$ is piecewise constant over $[n, n+1)$, where $n$ is an integer. Thus for $x \in[n, n+1)$

$$
\operatorname{Lim}_{\beta \rightarrow 1^{-}} P_{k}(n+\beta) \leqq P_{k}(x) \leqq P_{k}(n) .
$$

But

$$
\begin{aligned}
\operatorname{Lim}_{\beta \rightarrow 1^{-}} P_{k}(n+\beta) & =A_{k}(n)-V_{k}(n+1) \\
& =P_{k}(n)-\left(V_{k}(n+1)-V_{k}(n)\right) \\
& =P_{k}(n)+O\left(x^{k / 2-1}\right) .
\end{aligned}
$$

However, by (4), $\theta_{k} \geqq k / 2-1$. Therefore the sequence of "extreme" points $\left(N_{i},\left|P_{k}\left(N_{i}\right)\right|\right)$, defined such that $\left|P_{k}(n)\right|<\left|P_{k}\left(N_{i}\right)\right|$ for $n<N_{i+1}$, satisfies the first requirement of (5) for all $\theta$ of interest. This sequence is uniquely determined, given an initial element, and $N_{i+1}$ is the first integer for which $\left|P_{k}\left(N_{i}\right)\right|<$ $\left|P_{k}\left(N_{i+1}\right)\right|$.

For $x$ too large to calculate $P_{k}(x)$ conveniently for all integers, approximate extreme points can be chosen in the same manner as the true extreme points but from a more restricted set. These approximate extreme points are not necessarily a subset of the true extreme points. This later method, used by both Fraser and Gotlieb and Keller and Swenson (in a different context), is not so concise as the former but allows one to consider a larger range of $x$.

The present calculations on an IBM 7094 include $P_{k}(x)$ for $k=2,3,4,5,6$ and all integer $x \leqq 250,000\left(x^{1 / 2} \leqq 500\right)$; some 250 isolated values of $P_{2}(x)$ for $x^{1 / 2} \leqq$ $10,000,000$; and about 20 values of $P_{3}(x)$ for $x^{1 / 2} \leqq 9000$. The results of this work show that the calculated values follow the theoretical limits quite closely. The results for $k=2$ fail to indicate that $\theta_{2}$ is less than Chen Jing-ren's bound of $12 / 37=\overline{.324}$. For $k=3$ the most reasonable conclusion is $.5 \leqq \theta_{3} \leqq .6$.

Efficient algorithms for various combinations of $k$ and $x$ are presented in Section 2. Section 3 is composed of computing methods and Section 4 contains conclusions. 
2. Counting Algorithms. The most efficient method of evaluating $A_{k}(x)$ depends on the range of $k$ and $x$ and upon whether isolated values (for approximate extreme points) or a large number of consecutive values (for true extreme points) is desired. The following formula is similar to one given by Walfisz [1].

$$
\begin{aligned}
& A_{1}(0)=1, \\
& A_{1}(x)=A_{1}(x-1)+2 \delta(x)
\end{aligned}
$$

where

$$
\begin{aligned}
\delta(x) & = \begin{cases}1, & \text { for } x \text { a perfect square, } \\
0, & \text { otherwise, }\end{cases} \\
A_{k}(x) & =A_{k-1}(x)+2 \sum_{i=1}^{[\sqrt{x}]} A_{k-1}\left(x-i^{2}\right)
\end{aligned}
$$

Formula (8) provides the basic method of calculating $A_{k}(x)$. For large values of $x$, some terms of the above summation may be larger than the fixed-point singleword capacity of the computer $\left(2^{36}-1\right.$ on the IBM 7094). This difficulty can often be remedied by defining $R_{k}(x)$ as the number of points $\left(J_{1}, J_{2}, \cdots, J_{k}\right)$ such that

$$
\sum_{i=1}^{k} J_{i}^{2}=x
$$

It is evident that

$$
\begin{aligned}
& R_{k}(x)= \begin{cases}A_{k}(x)-A_{k}(x-1), & x \text { an integer, } \\
0, & \text { otherwise, }\end{cases} \\
& A_{k}(x)=\sum_{i=0}^{[x]} R_{k}(i) .
\end{aligned}
$$

Also

$$
\begin{aligned}
& R_{1}(0)=1 \\
& R_{1}(x)=2 \delta(x), \\
& R_{k}(x)=R_{k-1}(x)+2 \sum_{i=1}^{[\sqrt{x}]} R_{k-1}\left(x-i^{2}\right) .
\end{aligned}
$$

The similarities of (8) and (11) are noticeable. By changing initial values the same procedure may be used for either $A_{k}(x)$ or $R_{k}(x)$.

The next formula makes use of the symmetries involved in the set $L_{k}(x)$ resulting from permutations and negatives of ordered $k$-tuples. Define

$$
L_{k}^{\prime}(x)=\left\{\left(J_{1}, J_{2}, \cdots, J_{k}\right) \in L_{k}(x) \mid 0 \leqq J_{1} \leqq J_{2} \leqq \cdots \leqq J_{k}\right\}
$$

and let $M\left(J_{1}, J_{2}, \cdots, J_{k}\right)$ be the number of distinct permutations and negations of $J_{1}, J_{2}, \cdots, J_{k}$. Then we have

$$
M\left(J_{1}, J_{2}, \cdots, J_{k}\right)=\frac{2^{k-n(0)} k !}{\prod_{p=0}^{J_{k}} n(p) !} .
$$


where $n(p)$ is the number of $i$ for which $J_{i}=p$. Thus, if $\mathbf{Y}_{\mathbf{m}}=\left(J_{1}, J_{2}, \cdots J_{m}\right)$,

$$
A_{k}(x)=\sum_{\mathbf{Y}_{\mathbf{k}} \in L_{k}(x)} \quad 1=\sum_{\mathbf{Y}_{\mathbf{k}} \in L_{k^{\prime}}(x)} M\left(\mathbf{Y}_{\mathbf{k}}\right) .
$$

By rearranging (12) and (14) it follows that

$$
A_{k}(x)=1+\sum_{J_{k}=1}^{[\sqrt{x}]} \sum_{\mathbf{Y}_{\mathbf{k}-1} \in L_{k-1}^{\prime}\left(x-J_{k^{2}}\right) ; J_{k-1} \leqq J_{k}} M\left(\mathbf{Y}_{\mathbf{k}-1}, J_{k}\right) .
$$

Now, if $J_{k-1}<J_{k}$, then $M\left(J_{1}, J_{2}, \cdots, J_{k}\right)=2 k M\left(J_{1}, J_{2}, \cdots, J_{k-1}\right)$. Similarly, if $J_{k-i}<J_{k-i+1}=\cdots=J_{k}$, then

$$
M\left(J_{1}, J_{2}, \cdots, J_{k}\right)=2^{i}\left(\begin{array}{c}
k \\
i
\end{array}\right) M\left(J_{1}, J_{2}, \cdots, J_{k-i}\right) .
$$

Thus we have

$$
A_{k}(x)=1+\sum_{J_{k=1}=1}^{[\sqrt{x}]} \sum_{i=1}^{k} 2^{i}\left(\begin{array}{l}
k \\
i
\end{array}\right)_{\left.\mathbf{Y}_{\mathbf{k}-\mathbf{i}} \in L_{k-i}\left(x-i J_{k}\right)^{2}\right) ; J_{k-i}<J_{k}} M\left(\mathbf{Y}_{\mathbf{k}-\mathbf{i}}\right) .
$$

Now define

$$
S_{m}(Z, J)=\sum_{\mathbf{Y}_{\mathbf{m}} \in L_{m}^{\prime}(Z) ; J_{m}<J} M\left(\mathbf{Y}_{\mathbf{m}}\right)
$$

Thus

$$
A_{k}(x)=1+\sum_{J=1}^{[\sqrt{x}]} \sum_{i=1}^{k} 2^{i}\left(\begin{array}{l}
k \\
i
\end{array}\right) S_{k-i}\left(x-i J^{2}, J\right) .
$$

$S_{m}(Z, J)$ can be defined recursively as follows:

$$
\begin{aligned}
S_{m}(Z, J) & =1+\sum_{J_{m}=1}^{J-1} \sum_{i=1}^{m} 2^{i}\left(\begin{array}{c}
m \\
i
\end{array}\right) S_{m-i}\left(Z-i J_{m}^{2}, J_{m}\right), \\
S_{0}(Z, J) & = \begin{cases}1, & Z \geqq 0, \\
0, & Z<0 .\end{cases}
\end{aligned}
$$

It is convenient to note that

$$
\begin{aligned}
& S_{k}(x, \infty)=A_{k}(x), \\
& S_{k}(\infty, J)=(2 J-1)^{k} .
\end{aligned}
$$

Formula (18) used with (19) is the basic method for taking advantage of symmetries among the points of $L_{k}(x)$. By algebraic reduction the following general formula can be established:

$$
S_{m}(Z, J)=(2 N+1)^{m}+\sum_{i=1}^{m-1} \sum_{J_{m}=N+1}^{M_{N} i} 2^{i}\left(\begin{array}{c}
m \\
i
\end{array}\right) S_{m-i}\left(Z-i J_{m}^{2}, J_{m}\right)
$$

where $N=[\sqrt{Z / m}]$ and $\operatorname{MIN}_{i}=\min ([\sqrt{Z / i}], J-1)$. For complete generality $A_{k}(x)$ must be defined as in (19). For $k=2$ this simplifies to

$$
A_{2}(x)=1+4[\sqrt{x}]+4[\sqrt{x / 2}]^{2}+8 \sum_{J=[\sqrt{x / 2}]+1}^{[\sqrt{x}]}\left[\sqrt{x-J^{2}}\right] .
$$

This formula was known to Gauss. 
Formula (20) is of course ideally suited to programming for an algorithmic compiler; however, it can easily and efficiently be programmed in a machine-oriented language. Consequently it was coded in SCATRE for the 7094 and used for isolated values of $A_{k}(x)$ for large $x$.

One extension of (21) should be very valuable for computing isolated values of $A_{2}(x)$. It is possible, for certain $x$, to compute $A_{2}(x)$ for all $u$ subject to $|u|<2 \sqrt{2} x^{1 / 4}$ in nearly the same time necessary to compute $A_{2}(x+u)$ alone. For $x=10^{14}$, the largest argument used in this work, this would have made available over 17,000 results in about twice the time required for the single value. Needless to say, this is a significant improvement. Unfortunately, this method was not known when the computations were done for this paper, but the method has been used for moderate $x(x \approx 10,000)$.

Define the following:

$$
\begin{aligned}
x & =r^{2}+2 r=(r+1)^{2}-1, \quad r \text { an integer, } \\
U & \leqq 2 \sqrt{2 r}, \\
-U+2 & \leqq u \leqq U, \\
W & =\left[\sqrt{x-J^{2}}\right], \quad \text { where } J \text { is used in the context of (21). }
\end{aligned}
$$

The following theorem may be established by simple algebra:

$$
\left[\sqrt{x+u-J^{2}}\right]= \begin{cases}W+1, & u \geqq(W+1)^{2}-\left(x-J^{2}\right), \\ W-1, & u<W^{2}-\left(x-J^{2}\right), \\ W, & \text { otherwise. }\end{cases}
$$

Thus, if the remainder of the integer square root routine is available, it is easy to evaluate $\left[\sqrt{x+u-J^{2}}\right]$ in the process of applying (21).

The true value of this method lies in computing $A_{2}(x+u)$ for all suitable $u$ simultaneously. Define

$$
\begin{aligned}
& Q(0)=0, \\
& q(u)=0, \quad-U+2 \leqq u \leqq U .
\end{aligned}
$$

As $J$ runs from $[\sqrt{x / 2}]+1$ to $[\sqrt{x}]=r$, as in (21), do the following:

$$
\begin{aligned}
& Q(0)=q(0)+\left[\sqrt{x-J^{2}}\right]=Q(0)+W, \\
& q(v)=q(v)+1, \text { where } v=(W+1)^{2}-\left(x-J^{2}\right), \\
& q(v)=q(v)-1, \text { where } v=W^{2}-\left(x-J^{2}\right)-1 .
\end{aligned}
$$

Then, for $u>0$,

$$
\begin{aligned}
Q(u) & =Q(0)+\sum_{v=1}^{u} q(v)+[\sqrt{u-1}]-\sum_{J=[\sqrt{x / 2}]+1}^{[\sqrt{(x+u) / 2}]}\left[\sqrt{x+u-J^{2}}\right], \\
Q(-u) & =Q(0)+\sum_{v=1}^{u} q(-v)+\sum_{J=[\sqrt{(x-u) / 2}]+1}^{[\sqrt{x / 2}]}\left[\sqrt{x-u-J^{2}}\right] .
\end{aligned}
$$

And then, for all $u$,

$$
A_{2}(x+u)=1+4[\sqrt{x+u}]+4[\sqrt{(x+u) / 2}]^{2}+8 Q(u) .
$$


3. Computer Methods and Numerical Results. The time-consuming part of computing $A_{k}(x)$ by any of the methods mentioned in this paper is evaluating $[\sqrt{x}]$. However, from the logical order of summation successive arguments often happen to be close together. Furthermore, while most square root routines are floating-point, exact fixed-point results are necessary for this work. Thus the efficiency of the square root routine may be improved by using fixed-point operations and by using the previous result as a first approximation for the current argument. Using this and the identity

$$
(N \pm 1)^{2}=N^{2} \pm 2 N+1
$$

$A_{k}(x)$ may be calculated on a binary machine with no multiplications and no numbers larger than $x^{1 / 2}$ except the sum. This procedure was developed independently by Keller and Swenson [7] and the present author. It is particularly useful for employing (26). Keller and Swenson present the necessary algorithm and a basic derivation of the process.

For the current paper two methods were used for determining $A_{k}(x)$. For calculating isolated values, (20) and the above method were used. Special square root routines were used throughout. The time to compute $A_{k}(x)$ was on the order of

$$
T(k, x) \alpha \frac{x^{(k-1) / 2}}{k !} \text {. }
$$

When a large quantity of consecutive values was desired, (8) and (11) were used. An IBM 1301 Disc File was available for additional storage. This Disc File is particularly desirable in allowing the use of one portion of core-storage for computation while data is moved between the Disc File and another portion of core. For the present problem this effectively created a million words of core-storage. The time required to compute $A_{k}(x)$ for $2 \leqq k \leqq K$ and all integer $x \leqq X$ is

$$
T(K, X) \alpha(k-1) x^{3 / 2} \text {. }
$$

Using this method $3 \frac{1}{2}$ hours were required for $T(6,250000)$. Formula (11) was used with the assumption that $R_{k}(x)<2^{36}$. This assumption was violated near $R_{6}(40,000)$.

Integer arithmetic was used exclusively for $A_{k}(x)$ in all programs, and it is expected that all values are correct. Complete agreement was noted for all values published in [7]. Similar agreement existed with [4] except for $A_{3}\left(1800^{2}\right)$, the largest argument published in that paper. This value was calculated twice for this paper, each calculation requiring 3 minutes.

4. Conclusions. Table 1 gives the first fifty true extreme points for $k=2,3$. The number of extreme points for $x \leqq 250,000\left(x^{1 / 2} \leqq 500\right)$ is

$\begin{array}{ccc}k & \begin{array}{c}\text { number of } \\ \text { extreme points }\end{array} & \\ 2 & 76 & \\ 3 & 80 & \\ 4 & 170 & \\ 5 & 434 & (x<40,000) . \\ 6 & 474 & \end{array}$


TABle 1

First 50 extreme points for $k=2,3$

\begin{tabular}{|c|c|c|c|c|c|}
\hline$X_{i}$ & $A_{2}\left(X_{i}\right)$ & $P_{2}\left(X_{i}\right)$ & $X_{i}$ & $A_{3}\left(X_{i}\right)$ & $P_{3}\left(X_{i}\right)$ \\
\hline 1 & 5 & 2 & 1 & 7 & 3 \\
\hline 2 & 9 & 3 & 2 & 19 & 7 \\
\hline 5 & 21 & 5 & 5 & 57 & 10 \\
\hline 10 & 37 & 6 & 6 & 81 & 19 \\
\hline 20 & 69 & 6 & 14 & 251 & 32 \\
\hline 24 & 69 & -6 & 21 & 437 & 34 \\
\hline 26 & 89 & 7 & 29 & 691 & 37 \\
\hline 41 & 137 & 8 & 30 & 739 & 51 \\
\hline 53 & 177 & 10 & 54 & 1743 & 81 \\
\hline 130 & 421 & 13 & 90 & 3695 & 119 \\
\hline 149 & 481 & 13 & 134 & 6619 & 122 \\
\hline 205 & 657 & 13 & 155 & 8217 & 134 \\
\hline 234 & 749 & 14 & 174 & 9771 & 157 \\
\hline 287 & 885 & -17 & 230 & 14771 & 160 \\
\hline 340 & 1085 & 17 & 234 & 15155 & 161 \\
\hline 410 & 1305 & 17 & 251 & 16831 & 174 \\
\hline 425 & 1353 & 18. & 270 & 18805 & 221 \\
\hline 480 & 1489 & -19 & 342 & 26745 & 252 \\
\hline 586 & 1861 & 20 & 374 & 30551 & 254 \\
\hline 840 & 2617 & -22 & 461 & 41755 & 294 \\
\hline 850 & 2693 & 23 & 494 & 46297 & 305 \\
\hline 986 & 3125 & 27 & 550 & 54339 & 309 \\
\hline 1680 & 5249 & -29 & 666 & 72359 & 364 \\
\hline 1843 & 5761 & -29 & 750 & 86407 & 371 \\
\hline 2260 & 7129 & 29 & 810 & 96969 & 405 \\
\hline 2591 & 8109 & -31 & 990 & 131059 & 580 \\
\hline 3023 & 9465 & -32 & 1890 & 344859 & 682 \\
\hline 3024 & 9465 & -35 & 2070 & 395231 & 734 \\
\hline 3400 & 10717 & 36 & 2486 & 519963 & 756 \\
\hline 3959 & 12401 & -37 & 2757 & 607141 & 763 \\
\hline 3960 & 12401 & -40 & 2966 & 677397 & 776 \\
\hline 5182 & 16237 & -43 & 3150 & 741509 & 959 \\
\hline 5183 & 16237 & -46 & 3566 & 893019 & 1028 \\
\hline 7920 & 24833 & -48 & 3630 & 917217 & 1105 \\
\hline 9796 & 30725 & -50 & 4554 & 1288415 & 1120 \\
\hline 11233 & 35237 & -53 & 4829 & 1406811 & 1170 \\
\hline 14883 & 46701 & -55 & 5670 & 1789599 & 1205 \\
\hline 15119 & 47441 & -57 & 5750 & 1827927 & 1550 \\
\hline 15120 & 47441 & -60 & 8154 & 3085785 & 1570 \\
\hline 19593 & 61493 & -60 & 8382 & 3216051 & 1576 \\
\hline 21600 & 67797 & -61 & 8774 & 3444439 & 1851 \\
\hline 21603 & 67805 & -63 & 8910 & 3524869 & 1930 \\
\hline 21604 & 67805 & -66 & 10350 & 4412643 & 2028 \\
\hline 22177 & 69605 & -66 & 10710 & 4645127 & 2404 \\
\hline 28559 & 89653 & -68 & 15734 & 8269399 & 2411 \\
\hline 28560 & 89653 & -71 & 15750 & 8282167 & 2565 \\
\hline 31679 & 99449 & -74 & 16302 & 8721339 & 2675 \\
\hline 31680 & 99449 & -77 & 17550 & 9741669 & 2895 \\
\hline 38015 & 119349 & -79 & 23310 & 14910309 & 2905 \\
\hline 38016 & 119349 & -82 & 23894 & 15474065 & 2940 \\
\hline 38017 & 119349 & -85 & 24174 & 15746999 & 3133 \\
\hline
\end{tabular}


This reflects the increasing values of $\theta_{k}$ and perhaps more "regularity" for the higher values of $k$.

The problem of showing

$$
\operatorname{Limsup}_{i \rightarrow \infty} \frac{\left|P_{k}\left(N_{i}\right)\right|}{N_{i}{ }^{\theta}}<+\infty
$$

is equivalent to showing

$$
\operatorname{Limsup}_{i \rightarrow \infty}\left(\log \left|P_{k}\left(N_{i}\right)\right|-\theta \log N_{i}\right)<+\infty .
$$

Graphically this corresponds to finding a straight line with slope $\theta$ which majorizes the points $\left(\log N_{i}, \log \left|P_{k}\left(N_{i}\right)\right|\right)$. Figure 1 shows the sequence of extreme points for $x \leqq 250,000$. Only a sample of the points for $k=5,6$ are shown.

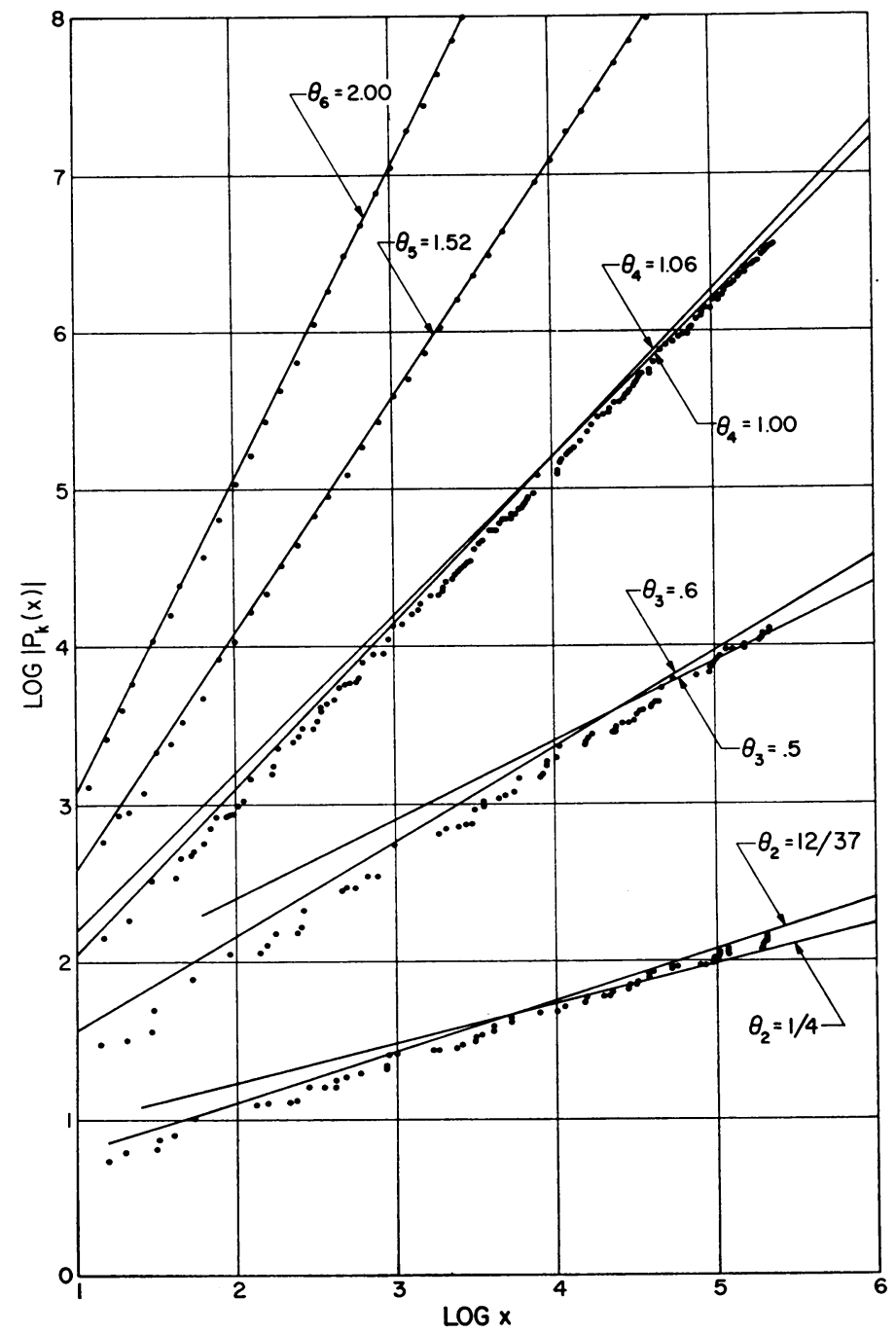

FIGURE 1. Extreme points for $x \leqq 250,000$ and $k=2,3,4,5,6$. 
TABLE 2

\begin{tabular}{r|r|r|r|r|r}
\hline \multicolumn{1}{c|}{$x^{1 / 2}$} & \multicolumn{1}{|c|}{$A_{2}(x)$} & \multicolumn{1}{|c|}{$P_{2}(x)$} & $x^{1 / 2}$ & \multicolumn{1}{|c}{$A_{3}(x)$} & \multicolumn{1}{|c}{$P_{3}(x)$} \\
1000000 & 3141592649625 & -3965 & 1000 & 4188781437 & -8768 \\
1500000 & 7068583465945 & -4632 & 1200 & 7238202017 & -27457 \\
2000000 & 12566370610285 & -4074 & 1400 & 11494026189 & -14133 \\
2500000 & 19634954076697 & -8239 & 1600 & 17157266213 & -18466 \\
3000000 & 28274333873841 & -8467 & 1800 & 24428982249 & -42225 \\
3500000 & 38484509999277 & -7198 & 2000 & 33510290993 & -30645 \\
4000000 & 50265482451357 & -6080 & 2500 & 65449818205 & -28745 \\
4500000 & 63617251226505 & -8688 & 3000 & 113097275709 & -59820 \\
5000000 & 78539816333093 & -6652 & 3500 & 179594325465 & -54565 \\
5500000 & 95033177762429 & -8662 & 4000 & 268082474393 & -98713 \\
6000000 & 113097335520185 & -9048 & 4500 & 381703453381 & -54030 \\
6500000 & 132732289606241 & -7928 & 5000 & 523598707861 & -67737 \\
7000000 & 153938040012805 & -13095 & 5500 & 696909887157 & -83164 \\
7500000 & 176714586754401 & -10025 & 6000 & 904778525345 & -158889 \\
8000000 & 201061929820913 & -8834 & 6500 & 1150346427953 & -82036 \\
8500000 & 226980069212125 & -9738 & 7000 & 1436754948853 & -91389 \\
9000000 & 254469004930845 & -9928 & 7500 & 1767145772565 & -95079 \\
9500000 & 283528736973257 & -13222 & 8000 & 2144660422929 & -161922 \\
9600000 & 289529178944573 & -10262 & 8500 & 2572440705977 & -78537 \\
9700000 & 295592452772029 & -4235 & 9000 & 3053627854381 & -204908 \\
9800000 & 301718558438929 & -11835 & & & \\
9900000 & 307907495964805 & -13531 & & & \\
10000000 & 314159265350589 & -8390 & & & \\
\hline
\end{tabular}

The lines drawn represent the minimum slopes which appear to parallel the extreme points. In addition, for $k=2,3,4$ the theoretical minima for $\theta_{k}$ (see (4)) are shown. If $\theta_{k}$ is estimated from these points, the results are

$$
\begin{aligned}
& \theta_{2} \risingdotseq . \overline{324}=12 / 37, \\
& \theta_{3} \risingdotseq .60=3 / 5, \\
& \theta_{4} \risingdotseq 1.06, \\
& \theta_{5} \risingdotseq 1.52, \\
& \theta_{6} \risingdotseq 2.00 .
\end{aligned}
$$

The accuracy of visual estimation limits this method to a precision of at most \pm .01 . For instance in Figure 1, for $k=2$ a line with slope $12 / 37$ would be indistinguishable from one with slope $1 / 3$. The way which the results for $k=4,5,6$ approach the known values suggests that this method is valuable for the range of $x$ used.

In addition to the true extreme points for $x^{1 / 2} \leqq 500$ a number of approximate extreme points were calculated from isolated values of $P_{2}(x)$ and $P_{3}(x)$. Some of these are shown in Table 2. In Figure 2, the values of $P_{2}(x)$ for $x^{1 / 2} \leqq 10,000,000$ are shown with the true extreme points for $x^{1 / 2} \leqq 500$. If only the approximate extreme points are considered, one is led to agree with Fraser and Gotlieb [4] that " $\theta_{2}=\frac{1}{4}$ is not inconsistent with observed results." But when the distribution of approximate extreme points is considered independently of sampling distribution, 


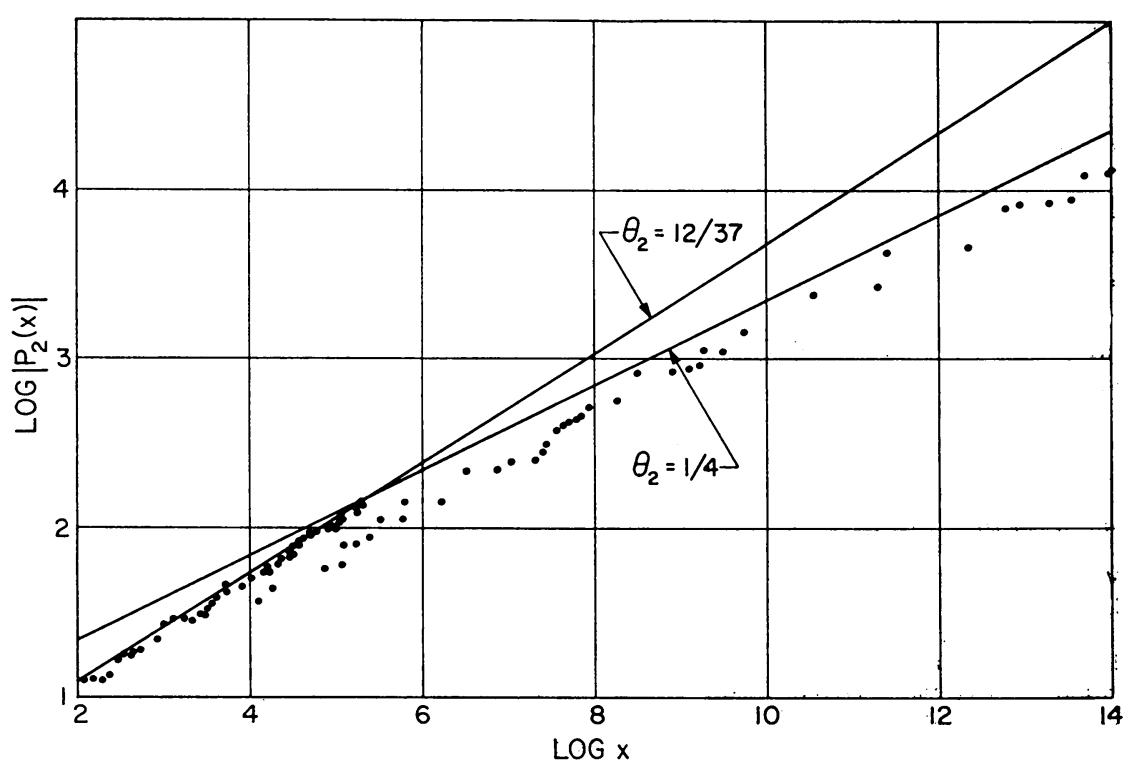

Figure 2. Distribution of true and approximate extreme points for $k=2$.

there is no reason to believe that the true extreme points do not continue near slope $12 / 37$ for $x^{1 / 2}>500$. Thus it is not reasonable to conjecture from these results that $\theta_{2}$ is appreciably less than Chen Jing-ren's bound of $12 / 37$. A logical conjecture based upon these results is $\theta_{3} \geqq .3$.

The approximate extreme points for $k=3$ suggest that $.5 \leqq \theta_{3} \leqq .6$, but there are too few points from which to extrapolate with assurance. For instance half of the isolated values qualify as approximate extreme points. The time required to calculate more values of $P_{3}(x)$ would be prohibitive.

An additional matter of interest is the sign of $P_{k}(x)$. Keller and Swenson reported that, while most of the values of $P_{2}(x)$ for integer values of $x^{1 / 2} \leqq 260,000$ were negative, the sign distribution for noninteger $x^{1 / 2}$ "was about uniform or perhaps even slightly biased in favor of positive values." In this experiment all of the true extreme values for $3400<x \leqq 250,000$ and all of the isolated values for integer $x^{1 / 2} \leqq 10,000,000$ were negative.

For $k=3,95 \%$ of the true extreme values were positive while the larger isolated values were negative. The four negative extreme values were among the larger extreme points.

For $k=4,5,6$ all of the true extreme values were positive.

Another question is whether or not noninteger values of $x$ would provide different extreme points than the integer values used thus far. From (6) we need only consider $\operatorname{Lim}_{\beta \rightarrow 1^{-}} P_{k}(n+\beta)$. This question is of little interest for $k=2$ because $P_{2}(n+\beta)=P_{2}(n)-\pi$. However for $k=3$, for $x<1000$, the $P_{3}(n+\beta)$ values were of the same magnitude as the $P_{3}(n)$ values. For the larger values of $k$ and necessarily smaller $x$, in accordance with the greater density of extreme points as in (27), there is an alternation of extreme points for small $x$ and a random assortment for larger $x$. 
5. Acknowledgements. The author would like to express his appreciation to Stephen Czuchlewski of Manhattan College (now at Yale University), J. R. Ehrman of the University of Illinois, and T. M. Apostol of the California Institute of Technology for assistance in this work.

Department of Computer Science

University of Illinois

Urbana, Illinois

Now at

California Institute of Technology

Pasadena, California

1. A. Walfisz, Gitterpunkte in mehrdimensionalen Kuglen, Monografie Matematyczne, Vol. 33, Panstwowe Wydawnictwo Naukowe, Warsaw, 1957. MR $20 * 3826$.

2. J. R. Wilton, "The lattice points of a circle: an historical account of the problem," Messenger of Mathematics, v. 58, 1929, pp. 67-80.

3. Chen Jing-Ren, Science Abstracts of China, v. 2, 1964, Number 2, abstract 271.

4. W. Fraser \& C. C. Gothes, "A calculation of the number of lattice points in the circle and sphere," Math. Comp., v. 16, 1962, pp. 282-290. MR 27 *5722.

5. Chen Jing-Ren, Science Abstracts of China, v. 2, 1964, Number 1, abstract 213.

6. H. L. Mrcheld, III, Numerical Experiments on the Number of Lattice Points in the Circle, Appl. Math. and Statist. Labs., Stanford Univ., Stanford, California, 1961.

7. H. B. Keller \& J. R. Swenson, "Experiments on the lattice problem of Gauss," Math. Comp., v. 17, 1963, pp. 223-230. MR $29 * 3445$. 